\title{
Fluorescence decay in aperiodic Frenkel lattices
}

\author{
Francisco Domínguez-Adame* and Enrique Maciá ${ }^{\dagger}$ \\ Departamento de Física de Materiales, Facultad de Físicas, Universidad Complutense, E-28040 Madrid, Spain
}

(Received 5 December 1995)

\begin{abstract}
We study motion and capture of excitons in self-similar linear systems in which interstitial traps are arranged according to an aperiodic sequence, focusing our attention on Fibonacci and Thue-Morse systems as canonical examples. The decay of the fluorescence intensity following a broadband pulse excitation is evaluated by solving the microscopic equations of motion of the Frenkel exciton problem. We find that the average decay is exponential and depends only on the concentration of traps and the trapping rate. In addition, we observe small-amplitude oscillations coming from the coupling between the low-lying mode and a few high-lying modes through the topology of the lattice. These oscillations are characteristic of each particular arrangement of traps and they are directly related to the Fourier transform of the underlying lattice. Our predictions then can be used to determine experimentally the ordering of traps. [S0163-1829(96)08019-8]
\end{abstract}

\section{INTRODUCTION}

The discovery of quasicrystals ${ }^{1}$ and the fabrication of aperiodic low-dimensional systems ${ }^{2}$ have encouraged the study of the physical properties of solids with long-range order, lacking translational symmetry. Physical aperiodic systems, whose structural order is described by means of deterministic sequences, present rather exotic electronic properties not shared by crystalline and amorphous solids. Peculiar signatures of these unique electronic states are Cantor-like spectra and self-similar wave functions. Several electronic properties of solids can be inferred from optical characterization techniques such as optical absorption, photoluminescence, and fluorescence after pulse excitation. Therefore, a complete understanding of the interplay between the electronic properties and the underlying aperiodic order requires a detailed analysis of the optical dynamics of this kind of ordering of matter. This interest has motivated various works dealing with optical properties of aperiodic systems, mainly devoted to Fibonacci and Thue-Morse semiconductor superlattices. ${ }^{3-6}$

Following a long term project regarding electronic and transport properties of aperiodic systems, ${ }^{7-10}$ our group focused its attention on optical absorption spectra of Frenkel excitons in Fibonacci and Thue-Morse lattices. ${ }^{11}$ Our main aim was to learn about the phenomenology of coherent exciton dynamics in molecular aggregates and polymers exhibiting long-range correlations. We considered Fibonacci and Thue-Morse lattices as canonical aperiodic models that are neither periodic nor random. In turn, they are inherently close to realistic aperiodic systems where the effects of longrange order might change dramatically the exciton dynamics. We found numerically several characteristic lines specific of each aperiodic system that are not present in the spectra of periodic or random Frenkel lattices, thus being an adequate way to determine the particular structural order of the system from experiments. ${ }^{11}$ Moreover, using a model based on the Pauli master equation, we have also studied motion and capture of incoherent excitons when traps are arranged according to aperiodic sequences. ${ }^{12}$ As an interesting feature, amenable to experimental confirmation by means of luminescence studies at moderate temperature, we obtained that the decay of the survival fraction of incoherent excitons is simply exponential, $P(t) \sim \exp (-A t)$, instead of the asymptotic stretched exponential $P(t) \sim \exp \left(-A t^{1 / 2}\right)$ appearing in one-dimensional random lattices. ${ }^{13}$ It is well known that the exponent of the stretched exponential varies when passing from trapping of classical (incoherent) to quantum (coherent) excitons in random lattices. ${ }^{14}$ Then a natural question arises in the context of aperiodic systems, namely, whether the simple exponential behavior of the survival fraction holds in the quantum case.

In this work we investigate the decay fluorescence due to Frenkel excitons in linear systems with interstitial traps arranged according to the Fibonacci and Thue-Morse sequences. We have focused on the analysis of aperiodic arrangements of traps in systems where optically active centers are placed in a straight line. Nevertheless, the model can also be applied to other possible geometric configurations of interest such as, for example, zigzag chains whose $s p^{2}$ hybridization is considered explicitly. To this end, we make use of a general treatment that allows us to study the dynamics of Frenkel excitons in these lattices, solve the microscopic equations of motion, and find the fluorescence intensity as a function of time. The main results of this paper are the following. First, we find out the decay law of the fluorescence intensity at low temperature, and compare it to the abovementioned simple exponential trend at moderate temperature. Second, and most important from an experimental point of view, we obtain distinctive features of the decay curves $P(t)$, allowing us to unveil the ordering of traps present in the system. By means of an analytical approach we are able to explain the origin of these characteristic oscillations, which are related to the Fourier transform of the arrangement of traps. Thus we successfully relate an optical property (fluorescence decay) with a structural one (topology of the lattice).

The remainder of the paper is organized as follows. In Sec. II we describe our model Hamiltonian and the way we arrange the traps following the Fibonacci and Thue-Morse sequences. In Sec. III we focus our attention on the fluorescence intensity following a broadband pulse excitation and solve the microscopic equation of motion. We find numeri- 
cally that, superimposed to an overall exponential decay, there exist small-amplitude oscillations characteristic of each particular system. In Sec. IV we develop a homogeneous medium approximation that accounts for the exponential decay, and, in Sec. V we introduce a perturbative treatment that successfully explains the small-amplitude oscillations. In this way, we clearly demonstrate that the frequency of the oscillations depends on the specific aperiodic order of the distribution of traps and, in addition, that these oscillations arise as a consequence of the coupling between the low-lying mode and few high-lying modes through the topology of the lattice. Section VI concludes the paper with a brief summary of results and some general remarks on their physical implications.

\section{PHYSICAL MODEL AND THEORY}

\section{A. Model Hamiltonian}

We consider a system of $N$ optically active two-level centers, occupying positions $\mathbf{r}_{n}$ on a linear regular lattice with spacing $a$. For our present purposes we neglect all thermal degrees of freedom. Therefore, the effective Frenkel Hamiltonian describing this system can be written as (we use units such that $\hbar=1)^{14-17}$

$$
\mathscr{H}=\sum_{n}\left(V_{n}-i \Gamma_{n}\right) a_{n}^{\dagger} a_{n}+\sum_{l \neq n} J_{n l} a_{n}^{\dagger} a_{l}
$$

Here $a_{n}^{\dagger}$ and $a_{n}$ are operators creating and annihilating an electronic excitation of energy $V_{n}$ at site $n$, respectively. $J_{n l}(n \neq l)$ is the intersite interaction of dipole origin between centers $n$ and $l$. In the case where all centers have equal and parallel dipole moment the interaction can be cast in the form $J_{n l}=-J\left(a /\left|\mathbf{r}_{n}-\mathbf{r}_{l}\right|\right)^{3}$, where $J$ is the coupling between nearest-neighbor centers. In this paper $J$ is the unit of energy and $J^{-1}$ the unit of time. Moreover, since $J_{n l}$ is a rapidly decreasing function of the distance between centers, we will omit interactions beyond nearest neighbors.

According to previous works, ${ }^{15-18}$ the non-Hermitian term $-i \Sigma_{n} \Gamma_{n} a_{n}^{\dagger} a_{n}$ accounts for the irreversible trapping of the exciton due to interstitial traps and the subsequent photon emission. Here $\Gamma_{n}=\Gamma$ if the $n$ center has a trap associated with it and $\Gamma_{n}=0$ otherwise, $\Gamma$ being the trapping rate. The site energy $V_{n}$ can be written, without loss of generality, as $\langle V\rangle+\Delta V_{n}$. The first term stands for the average excitation energy and we drop it by setting an appropriate reference. The second term is a random variable reflecting the offset energy due to the influence of the surrounding medium. Since we are mainly interested in the trapping dynamics, we neglect the effects of diagonal disorder $\left(\Delta V_{n}=0\right)$, noting that they could be easily incorporated in our treatment if necessary.

\section{B. Fibonacci lattice}

In this work we will be concerned with the Frenkel exciton dynamics and trapping processes in systems presenting an aperiodic distribution of interstitial traps. The Fibonacci lattice (FL) is the archetypal example of deterministic and quasiperiodically ordered structure. Any arbitrary Fibonacci system presents two kinds of building blocks. In our case, we choose those blocks as individual centers with and without a trap associated with it, hereafter called $T$ and $S$, respectively. The Fibonacci arrangement can be generated by the substitution rule $S \rightarrow S T, T \rightarrow S$. In this way, finite and self-similar aperiodic lattices are obtained by $n$ successive applications of the substitution rule. The $n$th generation lattice will have $N=F_{n}$ elements, where $F_{n}$ denotes a Fibonacci number. Such numbers are generated from the recurrence law $F_{n}=F_{n-1}+F_{n-2}$ starting with $F_{0}=F_{1}=1$; as $n$ increases the ratio $F_{n-1} / F_{n}$ converges toward $\tau=(\sqrt{5}-1) / 2$ $=0.618 \ldots$, an irrational number that is known as the inverse golden mean. Therefore, lattice sites are arranged according to the sequence STSSTSTS . . . . This type of distribution of traps will be referred to as minority lattice since the fraction of $T$ centers ( $1-\tau$ for large enough FL's) is smaller than the fraction of $S$ centers ( $\tau$ for large enough FL's). Moreover, it is worth noticing that $T$ centers appear isolated in the minority lattice. In the same way, one can generate the majority lattice by replacing $S \leftrightarrow T$, thus obtaining the sequence TSTTSTST . . . In this case $T$ centers can appear isolated or paired.

\section{Thue-Morse lattice}

The Thue-Morse lattice (TML) is also constructed from two building blocks $S$ and $T$. The substitution rule in this case is $S \rightarrow S T, T \rightarrow T S$ and the $n$th generation lattice contains $2^{n}$ centers. It is clear that the fraction of $T$ centers is exactly $1 / 2$ for any generation lattice. The resulting sequence STTSTSST . . is also aperiodic and self-similar but not quasiperiodic. Notice that both $S$ and $T$ centers appear isolated or paired. The TML present an inversion center so that the lattice is invariant under the transformation $S \leftrightarrow T$.

\section{Computation of physical magnitudes}

Having presented our model we now briefly describe the method we have used to calculate the fluorescence decay following a short-pulse excitation. The calculation involves the total dipole moment operator $\mathscr{D}=\Sigma_{n} d_{n}\left(a_{n}^{\dagger}+a_{n}\right)$, where $d_{n}$ is the dipole moment of the $n$ center. Notice that we are restricting ourselves to the case of systems whose length is much smaller than the optical wavelength. After the excitation, the state vector at time $t$ evolves according to $|\psi(t)\rangle=\exp (-i \mathscr{H} t) \mathscr{D}|\mathrm{vac}\rangle,|\mathrm{vac}\rangle$ being the exciton vacuum state. Besides radiative effects, the fluorescence intensity, normalized to the value at $t=0$, is given by ${ }^{16}$

$$
P(t)=\frac{|\langle\psi(0) \mid \psi(t)\rangle|^{2}}{|\langle\psi(0) \mid \psi(0)\rangle|^{2}}
$$

The fluorescence intensity can also be viewed as the probability of finding an exciton in the $\mathbf{k}=\mathbf{0}$ mode at time $t$.

A reliable method for determining $P(t)$ numerically has been given by Huber and Ching. ${ }^{16}$ These authors introduced a set of correlation functions,

$$
G_{n}(t)=\sum_{l} d_{l}\left\langle\operatorname{vac}\left|a_{n}(t) a_{l}^{\dagger}\right| \mathrm{vac}\right\rangle
$$


where $a_{n}(t)=\exp (i \mathscr{G} t) a_{n} \exp (-i \mathscr{H} b t)$ is the annihilation operator in the Heisenberg representation. The function $G_{n}(t)$ obeys the equation of motion

$$
i \frac{d G_{n}(t)}{d t}=\sum_{l} H_{n l} G_{l}(t),
$$

with the initial condition $G_{n}(0)=d_{n}$. The diagonal elements of the tridiagonal matrix $H_{n l}$ are $-i \Gamma_{n}$ whereas off-diagonal elements are simply given by $-J$. The microscopic equation of motion is a discrete Schrödinger-like equation on a lattice and standard numerical techniques may be applied to obtain the solution. Once these equations of motion are solved, the fluorescence intensity is evaluated from the relationship

$$
P(t)=\frac{\left|\sum_{n} d_{n}^{*} G_{n}(t)\right|^{2}}{\left.\left.\left|\sum_{n}\right| d_{n}\right|^{2}\right|^{2}} .
$$

\section{NUMERICAL RESULTS}

We have solved numerically the equation of motion (4) using an implicit (Crank-Nicholson) integration scheme. ${ }^{19}$ We note that energy is measured in units of $J$ and time in units of $J^{-1}$ and, since $4 J$ is the exciton bandwidth, the energy and time scales can be deduced from each particular experimental situation. Aperiodic lattices are generated using the inflation rules discussed above. In order to minimize end effects, spatial periodic boundary conditions are introduced in all cases. The maximum integration time and the integration time step were 20 and $2 \times 10^{-3}$, respectively. Smaller time steps give essentially the same results. In most cases of practical interest, one finds that the trapping rate is much less than the exciton bandwidth, that is, $\Gamma \ll 4 J$. Thus, as typical values, we have studied lattices with $\Gamma / J=0.01, \ldots, 0.05$. The maximum lattice size under consideration was $N=F_{12}=233$ for FL's and $N=2^{8}=256$ for TML's. Finally, local dipole moments are taken to be $d_{n}=1 / \sqrt{N}$.

In our numerical simulations we have found that the fluorescence intensity decays exponentially, for both FL's and TML's, in the full time interval considered by us. Figure 1 shows the results for majority and minority FL's with $N=233$ and TML's with $N=256$, but we have checked that this behavior is independent of the system size. As expected, the larger the trapping rate, the faster the decay of the fluorescence intensity. Moreover, for the same value of the trapping rate, this decay is faster on increasing the fraction of traps (minority FL's, TML's, and majority FL's). Hence, as occurs in the case of completely incoherent excitons, ${ }^{18}$ the decay of Frenkel excitons in FL's and TML's resembles that of periodic lattices instead of random ones, where the decay is stretched exponential. The average decay of the fluorescence intensity fits very well to an exponential of the form $\exp (-2 c \Gamma t)$ in the considered time interval, $c$ being the fraction of traps in each lattice. This time dependence has also been confirmed by fitting the data to stretched exponentials of the form $\exp \left(-A t^{\beta}\right)$, obtaining in all cases $\beta=1.0000 \pm 10^{-4}$

A detailed analysis of data plotted in Fig. 1 shows the occurrence of small-amplitude oscillations superimposed to the average exponential decay of the fluorescence intensity.

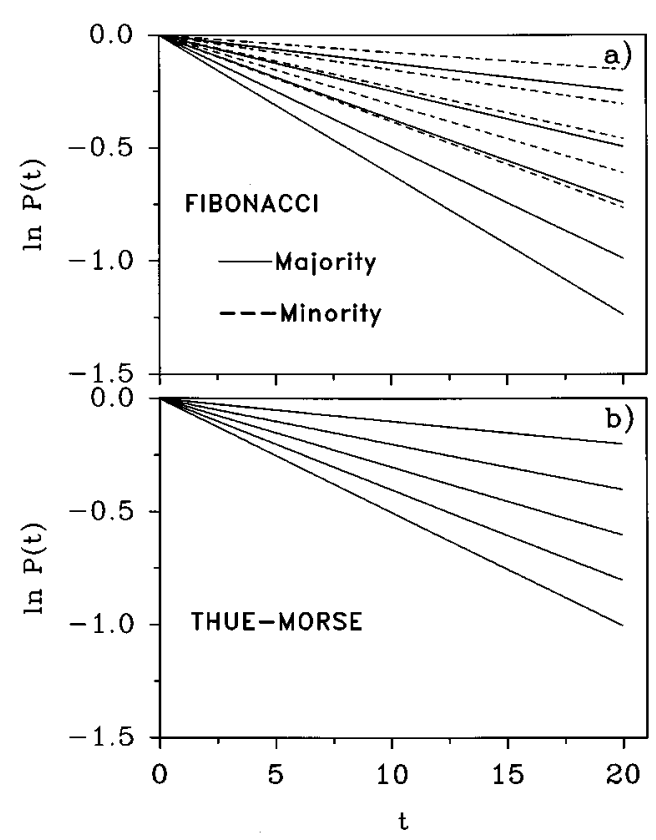

FIG. 1. Time decay of fluorescence intensity in (a) Fibonacci lattices with $N=F_{12}=233$ centers and a fraction of traps $c=0.618 \ldots$ (majority lattice, solid lines) and $c=0.382 \ldots$ (minority lattice, dashed lines), and (b) Thue-Morse lattices with $N=2^{8}=256$ centers. In all cases the trapping rate is, from top to bottom, $\Gamma=0.01, \ldots, 0.05$ in units of $J$.

The functional form $P(t)$ in all cases considered can be expressed as

$$
P(t)=\exp (-2 c \Gamma t)\left[1+\Gamma^{2} p(t)\right],
$$

where $p(t)$ is an oscillatory function which is independent of the trapping rate. This function can be readily extracted from numerical data as $p(t) \simeq \Gamma^{-2} \ln [P(t) \exp (2 c \Gamma t)]$. Figure 2 shows the typical form of the $p(t)$ function for any one of the trapping rates $\Gamma$ considered in our study. Remarkably, this function is, on one side, independent of the number of active centers present in the lattice and, most interestingly, it does not depend on the concentration of traps; i.e., it is exactly the same for both majority and minority versions of FL's-this invariance is, of course, trivial for TML's. Finally, by comparing Figs. 2(a) and 2(b) we realize that the oscillatory pattern of $p(t)$ is characteristic of the arrangement of traps, in the sense that its general features are very sensitive to the kind of aperiodic order being considered.

\section{HOMOGENEOUS MEDIUM APPROXIMATION}

In this section we develop a theoretical approach to explain the overall exponential decay of the fluorescence in aperiodic lattices. To this end we rewrite the system Hamiltonian $\mathscr{H}_{b}=\mathscr{H}_{\mathrm{HM}}-i \Gamma \mathscr{H}_{T}$, where

$$
\begin{gathered}
\mathscr{B}_{\mathrm{HM}}=-i c \Gamma \sum_{n} a_{n}^{\dagger} a_{n}-J \sum_{n}\left(a_{n}^{\dagger} a_{n+1}+a_{n+1}^{\dagger} a_{n}\right), \\
\mathscr{H}_{T}=\sum_{n}\left(\Gamma_{n} / \Gamma-c\right) a_{n}^{\dagger} a_{n} .
\end{gathered}
$$




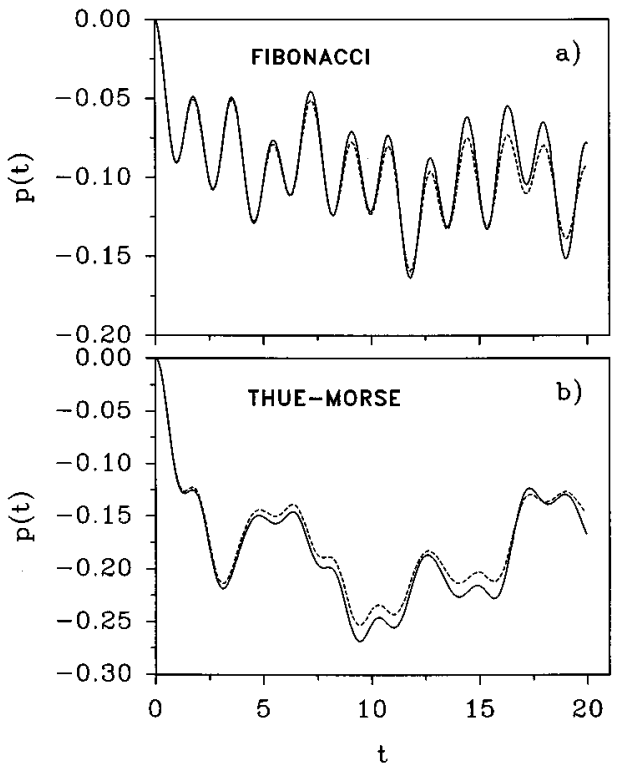

FIG. 2. Plot of $p(t) \simeq \Gamma^{-2} \ln [P(t) \exp (2 c \Gamma t)]$ as a function of time for (a) Fibonacci and (b) Thue-Morse lattice. Solid and dashed lines indicate numerical results and perturbative prediction, respectively. Results are independent of the number of lattice sites and the concentration of traps.

As a first approximation, we neglect the topology of the lattice and take $\mathscr{H} \simeq \mathscr{H}_{\mathrm{HM}}$. Physically this implies replacing the aperiodic distribution of traps by a uniform distribution with a renormalized trapping rate $c \Gamma$. To proceed we notice that the overlap between the state vector at time $t$ and the initial state vector may be calculated from ${ }^{20}$

$$
\langle\psi(0) \mid \psi(t)\rangle=\sum_{k}|\langle k|\mathscr{D}| \mathrm{vac}\rangle|^{2} \exp \left(-i E_{k} t\right),
$$

where $|k\rangle$ denotes the one-exciton eigenvectors of (7a) and $E_{k}$ the corresponding eigenvalues. The Hamiltonian $\mathscr{H}_{\mathrm{HM}}$ with periodic boundary conditions can be exactly diagonalized, yielding the eigenvectors ${ }^{21}$

$$
|k\rangle=\left(\frac{1}{N}\right)^{1 / 2} \sum_{n} \exp \left(2 \pi i \frac{n k}{N}\right) a_{n}^{\dagger}|\mathrm{vac}\rangle,
$$

with $k=0, \ldots, N-1$. The corresponding eigenvalues read

$$
E_{k}=-i c \Gamma-2 J \cos \left(2 \pi \frac{k}{N}\right) \equiv-i c \Gamma+\omega_{k}
$$

Therefore, the dipole moment matrix elements in the homogeneous medium approach satisfy the relation $\langle k|\mathscr{D}| \mathrm{vac}\rangle=\delta_{k 0} . \quad$ Consequently one gets $\langle\psi(0) \mid \psi(t)\rangle=\exp \left(-c \Gamma t-i \omega_{k} t\right)$ so that we obtain the exponential behavior $P_{\mathrm{HM}}(t)=\exp (-2 c \Gamma t)$. This result shows that, within the tight-binding approximation, the overall exponential decay of the fluorescence is a general property of ordered systems described in terms of the Hamiltonian (1), with independence of this order being periodic or aperiodic. We have confirmed this point by considering periodic arrangements of traps with different basis. In all cases we have observed that the exponential behavior predicted by the homogeneous medium approximation treatment successfully describes the overall decay obtained by numerical simulations. On the other hand, the exact result obtained within that approximation provides us with a starting point that will allow us to explain in full detail the small-amplitude oscillations shown in Fig. 2.

\section{PERTURBATIVE APPROXIMATION}

The replacement of a given aperiodic distribution of traps by a uniform one with a renormalized trapping rate is a crude representation of the real system. To develop a better approximation one must explicitly consider the effects of the topology on the exciton decay through the term $-i \Gamma \mathscr{H}_{T}$. To carry out such an approximation we have been inspired by the nondegenerate perturbation theory developed by Huber to study random systems with substitutional traps. ${ }^{20}$ Let $|\widetilde{k}\rangle$ be the perturbed eigenvector describing the exciton state when the influence of the term $-i \Gamma \mathscr{H}_{T}$ is taken into account. To evaluate the perturbed dipole moment matrix element, we expand $|\widetilde{k}\rangle$ in the basis of the unperturbed eigenvectors $|k\rangle$ given in $(9 \mathrm{a})$,

$$
|\widetilde{k}\rangle=|k\rangle-i \Gamma \sum_{l \neq k} \frac{\left\langle l\left|\mathscr{H}_{T}\right| k\right\rangle}{E_{k}-E_{l}}|l\rangle,
$$

whereas the perturbed eigenvalues are given by

$$
\widetilde{E_{k}}=E_{k}-i \Gamma\left\langle k\left|\mathscr{H}_{T}\right| k\right\rangle,
$$

with $E_{k}$ being the unperturbed eigenvalues. Making use of the fact that the dipole moment matrix element involving unperturbed eigenvectors is $\langle k|\mathscr{D}| \mathrm{vac}\rangle=\delta_{k 0}$, the overlap between $|\psi(0)\rangle$ and $|\psi(t)\rangle$ can be readily found, leading to

$$
\begin{aligned}
P(t) & =P_{\mathrm{HM}}(t)\left|\frac{1+\Gamma^{2} z(t)}{1+\Gamma^{2} z(0)}\right|^{2} \\
& =P_{\mathrm{HM}}(t)\left\{1+2 \Gamma^{2} \operatorname{Re}[z(t)-z(0)]\right\}+O\left(\Gamma^{4}\right),
\end{aligned}
$$

with

$$
z(t) \equiv \sum_{k \neq 0} \frac{\left|\left\langle 0\left|\mathscr{H}_{T}\right| k\right\rangle\right|^{2}}{\omega_{k 0}^{2}} \exp \left(-i \omega_{k 0} t\right),
$$

where we have defined $\omega_{k 0} \equiv \omega_{k}-\omega_{0}=4 J \sin ^{2}(\pi k / N)$. To obtain the above result we have made use of the result $\left\langle k\left|\mathscr{H}_{T}\right| k\right\rangle=0$, which according to (10b) indicates that $\widetilde{E}_{k}=E_{k}$ in the first-order perturbative treatment. Hence, recalling Eq. (6), one gets

$$
p(t)=2 \sum_{k \neq 0} \frac{\left|\left\langle 0\left|\mathscr{H}_{T}\right| k\right\rangle\right|^{2}}{\omega_{k 0}^{2}}\left(\cos \omega_{k 0} t-1\right) .
$$

The most remarkable fact of this expression is that it relates the small-amplitude oscillations of the fluorescence decay, an experimentally measurable magnitude, with the Fourier transform of the distribution of traps describing the topological ordering of the system. To demonstrate this point, we recall that unperturbed eigenvectors are orthogonal. Thus

$$
\begin{gathered}
\left\langle 0\left|\mathscr{G}_{T}\right| k\right\rangle=\left\langle 0\left|\sum_{n} \frac{\Gamma_{n}}{\Gamma} a_{n}^{\dagger} a_{n}\right| k\right\rangle=\frac{1}{N} \sum_{n} \frac{\Gamma_{n}}{\Gamma} \exp \left(2 \pi i \frac{k n}{N}\right), \\
k \neq 0 .
\end{gathered}
$$


which is nothing but the above-mentioned Fourier transform. A comparison of the perturbative prediction (13) with the numerical result is given in Fig. 2, where an excellent agreement is achieved for both Fibonacci and Thue-Morse aperiodic arrangements. At this point we should stress that the above result is valid for any arbitrary lattice. It relates the Fourier pattern of the distribution of traps with the finer details of the fluorescence decay. Since each particular arrangement of traps leads to a different Fourier pattern of $p(t)$, we arrive at the conclusion that the small-amplitude oscillations characterize every distribution of traps in much the same way that the usual $\mathrm{x}$-ray diffraction analysis characterizes the distribution of scattering centers in the space. It is also worth mentioning that it is not difficult to show from (14) that dual lattices, namely, those lattices obtained from the replacement $T \leftrightarrow S$, present the same value of $\left|\left\langle 0\left|\mathscr{H}_{T}\right| k\right\rangle\right|^{2}$. In particular, minority and majority Fibonacci lattices should display exactly the same oscillations, as is indeed the case in our numerical results.

In order to get a deeper insight into the small-amplitude oscillations in the case of aperiodic arrangement of traps, we relate our results with those reported in the various works dealing with the Fourier transform of these sequences, most of them in connection with x-ray diffraction and Raman scattering in aperiodic semiconductor superlattices, and neutron scattering in quasicrystals. ${ }^{22-26}$ Let us consider, in the first place, the case of the FL, which is known to exhibit a pure point Fourier spectrum displaying well-defined Bragg peaks. $^{25}$ The Fourier intensity $\left|\left\langle 0\left|\mathscr{H}_{T}\right| k\right\rangle\right|^{2}$ of FL's consists of a series of peaks located at values of the momentum $2 \pi(k / N)$ of the form $2 \pi(m+n \sigma)$, where $m$ and $n$ are two arbitrary integers and $\sigma \equiv(\sqrt{5}+1) / 2$ is the golden mean. Each peak of the Fourier intensity leads to a large contribution to the sum (13) and, consequently, to a well-defined frequency in the oscillatory pattern of $p(t)$. A simple inspection of Fig. 2(a) indicates that the pattern of $p(t)$ is dominated by a single frequency and, in fact, the Fourier analysis of the data gives the approximate value $\sim 3.456$ for this main frequency. The frequency of the particular mode $k_{p}$ that contributes significantly to the oscillatory pattern is then given by $\omega_{k_{p}}-\omega_{0}=3.456$, leading to $k_{p} / N \simeq 0.3798$, the ratio being independent of the system size. Therefore the mode is located close to the top of the excitonic band $(k / N=0.5)$. Figure 3(a) shows $\left|\left\langle 0\left|\mathscr{H}_{T}\right| k\right\rangle\right|^{2} / \omega_{k 0}^{2}$ as a function of the ratio $k / N$ evaluated from expression (14), the results being independent of the system size. A sequence of Bragg peaks, arranged according to successive powers of $\tau$, are clearly observed in this plot. The stronger one, which is responsible for the highest-frequency oscillations, is located at $k / N \simeq 0.38 \simeq \tau^{2}$. Making use of the relationships $\tau^{2}+\tau=1$ and $\sigma=1+\tau$, we find the two indices labeling this peak to be $m=2$ and $n=-1$. The Fourier pattern is symmetric around the top of the excitonic band and, in fact, there exists also a similar peak located at $k / N \simeq 0.62 \simeq 1-\tau^{2}=\tau$, whose indices are $m=-1$ and $n=1$. The remaining, in general weaker, peaks also give rise to oscillations in $p(t)$ but their contribution is not appreciable in the decay curve because they are closer to the bottom of the excitonic band and then lead to low-frequency oscillations.
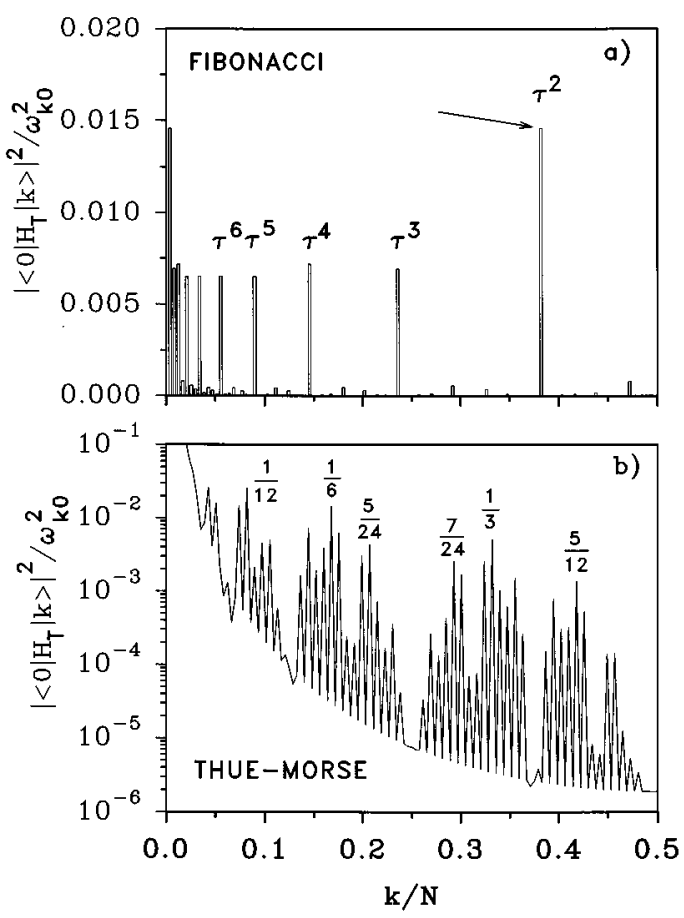

FIG. 3. (a) Plot of $\left|\left\langle 0\left|\mathscr{H}_{T}\right| k\right\rangle\right|^{2} / \omega_{k 0}^{2}$ as a function of the ratio $k / N$ for FL's showing the occurrence of well-defined Bragg peaks, labeled according to successive powers of the inverse golden mean $\tau$. The arrow indicates the peak responsible of the largest-frequency oscillations observed in Fig. 2. (b) Plot of $\left|\left\langle 0\left|\mathscr{H}_{T}\right| k\right\rangle\right|^{2} / \omega_{k 0}^{2}$ as a function of the ratio $k / N$ for a TML with $2^{8}=256$ active centers. A dense arrangement of peaks labeled according to the formula $(2 n+1) /\left(3 \times 2^{m}\right)$ is clearly observed.

Let us consider now the case of the TML, which is known to exhibit a singular continuous Fourier transform, lacking well-defined Bragg peaks. ${ }^{26}$ For finite TML's the Fourier intensity consists of a dense arrangement of peaks whose number depends on the sample size. For a TML composed of $2^{n}$ building blocks of the same size one expects to find $2^{n-1}$ peaks in the related Fourier transform whose positions are located at values of the momentum $2 \pi(k / N)$ of the form $(2 n+1) /\left(3 \times 2^{m}\right)$, where $n$ and $m$ are two arbitrary integers. ${ }^{26}$ An inspection of Fig. 2(b) indicates that, conversely to the case of FL, the pattern of the oscillatory fluorescence decay corresponding to the TML cannot be described in terms of a single main frequency. In fact, the Fourier analysis of numerical data shows a considerable number of frequencies closely packed. Figure 3(b) shows $\left|\left\langle 0\left|\mathscr{H}_{T}\right| k\right\rangle\right|^{2} / \omega_{k 0}^{2}$ as a function of the ratio $k / N$ for a TML with $N=2^{8}$ active centers. A dense distribution of peaks is clearly observed. We have checked that most peaks can be labeled by means of the formula $(2 n+1) /\left(3 \times 2^{m}\right)$. This we show in the figure for the most relevant peaks. By comparing Fig. 3(b) with X-ray results obtained for semiconducting TM superlattices, ${ }^{26}$ we see that the study of the oscillations in the fluorescence decay allows us to analyze the topological structure for a wide variety of aperiodic systems, even if those systems do not give rise to well-defined Bragg peaks in the reciprocal space.

Our results are suitable for a direct physical interpretation. Small-amplitude oscillations of largest frequency $\omega_{k_{p}}-\omega_{0}$ 
are caused by the coupling of two modes, namely, the lowest-lying and $k_{p}$, through the topology of the aperiodic distribution of traps. Notice that different arrangement of traps would lead to completely different Fourier intensity $\left|\left\langle 0\left|\mathscr{H}_{T}\right| k\right\rangle\right|^{2}$ and $p(t)$, as can be seen from a comparison of Figs. 2(a) and 2(b). Thus, the exciton acts as a probe of the spatial distribution of traps. This is one of the main results of the present work since it provides us with a useful method to be applied in experimental situations. To elucidate the particular arrangement of traps in a linear lattice, first we can analyze the fluorescence decay after $\delta$-pulse excitation. If this decay is stretched exponential, the arrangement of traps is completely disordered, whereas simple exponential behavior indicates absence of disorder corresponding to either periodic or aperiodic distribution of trapping centers. The slope of the average exponential decay permits one to find out the concentration of traps through the exponent value of $P_{\mathrm{HM}}(t)$, and then $p(t) \sim \ln \left[P(t) / P_{\mathrm{HM}}(t)\right]$. By performing the Fourier analysis of $p(t)$ one can determine its related power spectrum and then, comparing the obtained results with theoretical predictions from (13) and (14), to extract relevant information about the topological order of the underlying structure.

On the other hand, from a theoretical point of view, this work represents a twofold extension of the mathematical treatment originally introduced by Huber. ${ }^{20}$ First, instead of studying the asymptotic fluorescence decay in random Frenkel lattices we consider the case of general aperiodic systems. Second, while Huber evaluated the dipole moment matrix elements using unperturbed eigenvectors and perturbed eigenvalues, we show that this approach can only explain the occurrence of long-period oscillations. To account for shortperiod oscillations we must include the second term of (10a), that is, perturbed eigenvectors. This second term is responsible for the coupling between the lowest-lying mode and the high-lying modes through the aperiodic arrangement of traps, causing the short-period oscillations.

\section{CONCLUSIONS}

In this paper we have carried out a detailed analysis of the fluorescence decay in self-similar Frenkel lattices with interstitial traps arranged according to the Fibonacci and ThueMorse sequences. To this end, we have solved numerically the equation of motion of Frenkel excitons in the time domain. Unlike what was previously found in random lattices, we have observed that the fluorescence decay after $\delta$-pulse excitation, in which all centers are initially excited, behaves exponentially on average in the case of aperiodic systems. We have demonstrated that such a time decay is quite well described within a homogeneous medium approximation, where the aperiodic distribution of traps is replaced by a uniform distribution one with a renormalized trapping rate. This exponential trend is similar to that found in the case of incoherent transport through aperiodic lattices and, in spite of its dependence on the concentration of traps, it cannot be used to fully characterize each particular arrangement of trapping centers. Instead, quantum effects reveal themselves through small-amplitude oscillations superimposed to the average exponential decay. Interestingly, we have shown that the oscillatory pattern is characteristic of each particular lattice and, consequently, it can be considered as a fingerprint that allows us to elucidate the kind of underlying ordering present in the distribution of traps.

We have carried out a perturbation approach to account for the small-amplitude oscillations, finding excellent agreement with numerical data. The perturbative analysis clearly indicates that the oscillatory pattern of the fluorescence decay is directly related to the Fourier transform of the distribution of traps and, as a consequence, it is characteristic of the ordering present in the considered system. In this sense, we realize that the necessary condition for an arbitrary system to exhibit Fourier peaks is the occurrence of long-range order in its structure. This condition plays a major role in our theoretical analysis. Since each well-defined frequency contributing to the function $p(t)$ can be related to a distinctive peak in the Fourier transform of the lattice, the Fourier analysis of the time-dependent signal gives us direct information concerning the spatial distribution of traps. The physical origin of the fluorescence oscillations can be traced back to the coupling between the low-lying exciton mode and certain particular high-lying modes through the lattice topology. This means that excitons act as a probe of the spatial distribution of trapping centers. Hence the detailed analysis of time decay of the fluorescence, which uses excitons as a physical probe of the long-range order, represents a powerful tool to investigate the topology of general aperiodic systems from experiments, opening in this way an experimental technique that may be tentatively referred to as laser crystallography.

\section{ACKNOWLEDGMENTS}

The authors thank A. Sánchez and V. Malyshev for a critical reading of the manuscript. This work was supported by CICYT (Spain) through Project No. MAT95-0325.
*Electronic address: adame@valbuena.fis.ucm.es

†Also at Instituto de Estudios Interdisciplinares, El Guijo, Z4 Galapagar, E-28260 Madrid, Spain. Electronic address:

fimat04@emducms1.sis.ucm.es

${ }^{1}$ D. Shechtman, I. Blech, D. Gratias, and J. W. Cahn, Phys. Rev. Lett. 53, 1951 (1984); A. I. Goldman and R. F. Kelton, Rev. Mod. Phys. 65, 213 (1993).

${ }^{2}$ R. Merlin, K. Bajema, R. Clarke, F. Y. Juang, and P. K. Bhattacharya, Phys. Rev. Lett. 55, 1768 (1985).

${ }^{3}$ S.-R. Eric and S. Das Sarma, Phys. Rev. B 37, 4007 (1988).

${ }^{4}$ A. A. Yamaguchi, T. Saiki, T. Tada, T. Ninoyima, K. Misawa, and T. Kobayashi, Solid State Commun. 85, 223 (1993).

${ }^{5}$ D. Tuet, M. Potemski, Y. Y. Wang, J. C. Maan, L. Tapfer, and K. Ploog, Phys. Rev. Lett. 66, 2128 (1991).

${ }^{6}$ D. Munzar, L. Bocáek, J. Humlícek, and J. Ploog, J. Phys. Condens. Matter 6, 4107 (1994).

${ }^{7}$ F. Domínguez-Adame and A. Sánchez, Phys. Lett. A 159, 153 (1991).

${ }^{8}$ E. Maciá, F. Domínguez-Adame, and A. Sánchez, Phys. Rev. B 49, 9503 (1994).

${ }^{9}$ E. Maciá, F. Domínguez-Adame, and A. Sánchez, Phys. Rev. E 50, 679 (1994). 
${ }^{10}$ F. Domínguez-Adame, E. Maciá, B. Méndez, C. L. Roy, and A. Khan, Semicond. Sci. Technol. 10, 797 (1995).

${ }^{11}$ E. Maciá and F. Domínguez-Adame, Phys. Rev. B 50, 16856 (1994).

${ }^{12}$ F. Domínguez-Adame, E. Maciá, and A. Sánchez, Phys. Rev. B 51, 878 (1994).

${ }^{13}$ A. Sánchez, F. Domínguez-Adame, and E. Maciá, Phys. Rev. B 51, 173 (1995); F. Domínguez-Adame, M. A. Rodríguez, and A. Sánchez (unpublished).

${ }^{14}$ P. E. Parris, Phys. Rev. B 40, 4928 (1989).

${ }^{15}$ P. E. Parris, Phys. Rev. Lett. 62, 1392 (1989).

${ }^{16}$ D. L. Huber and W. Y. Ching, Phys. Rev. B 42, 7718 (1990).

${ }^{17}$ Th. Wagersreiter and H. F. Kauffmann, Phys. Rev. B 50, 9102 (1994).

${ }^{18}$ F. Domínguez-Adame, M. Méndez, A. Sánchez, and E. Maciá, Phys. Rev. B 49, 3839 (1994).
${ }^{19}$ W. H. Press, S. A. Teukolsky, W. T. Vetterling, and B. P. Flannery, Numerical Recipes in $C$, 2nd ed. (Cambridge University Press, New York, 1992).

${ }^{20}$ D. L. Huber, Phys. Rev. B 45, 8947 (1992).

${ }^{21}$ H. Fidder, J. Knoester, and D. A. Wiersma, J. Chem. Phys. 95, 7880 (1991).

${ }^{22}$ R. Merlin, IEEE J. Quantum Electron. 24, 1791 (1988), and references therein.

${ }^{23}$ C. Benoit, G. Poussigue, and A. Azougarh, J. Phys. Condens. Matter 2, 2519 (1990).

${ }^{24}$ C. Godrèche and J. M. Luck, J. Phys. A 23, 3769 (1990).

${ }^{25}$ J. Todd, R. Merlin, R. Clarke, K. M. Mohanty, and J. D. Axe, Phys. Rev. Lett. 57, 1157 (1986).

${ }^{26}$ F. Axel and H. Terauchi, Phys. Rev. Lett. 66, 2223 (1991); 73, 1308 (1994); M. Ishida, K. Kamigaki, T. Morioka, H. Kato, N. Sano, and H. Terauchi, J. Phys. Soc. Jpn. 61, 149 (1992). 